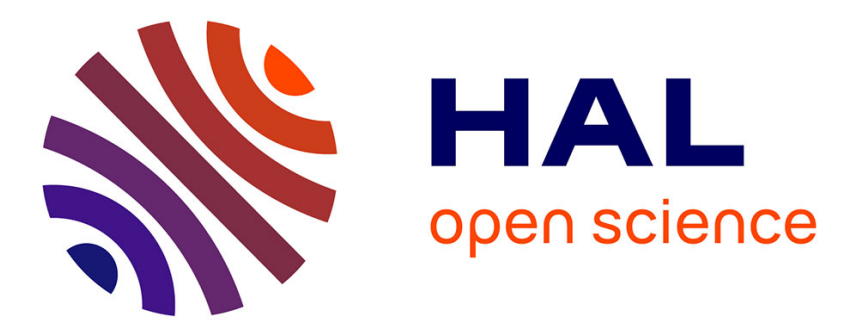

\title{
Etude comparative de différents dispositifs permettant de transformer un faisceau laser de puissance avec une répartition énergétique gaussienne en une répartition uniforme
}

\author{
J.C. Li, J. Merlin, J. Perez
}

\section{To cite this version:}

J.C. Li, J. Merlin, J. Perez. Etude comparative de différents dispositifs permettant de transformer un faisceau laser de puissance avec une répartition énergétique gaussienne en une répartition uniforme. Revue de Physique Appliquée, 1986, 21 (7), pp.425-433. 10.1051/rphysap:01986002107042500 . jpa00245460

\author{
HAL Id: jpa-00245460 \\ https://hal.science/jpa-00245460
}

Submitted on 1 Jan 1986

HAL is a multi-disciplinary open access archive for the deposit and dissemination of scientific research documents, whether they are published or not. The documents may come from teaching and research institutions in France or abroad, or from public or private research centers.
L'archive ouverte pluridisciplinaire HAL, est destinée au dépôt et à la diffusion de documents scientifiques de niveau recherche, publiés ou non, émanant des établissements d'enseignement et de recherche français ou étrangers, des laboratoires publics ou privés. 


\title{
Etude comparative de différents dispositifs permettant de transformer un faisceau laser de puissance avec une répartition énergétique gaussienne en une répartition uniforme
}

\author{
J. C. $\operatorname{Li}\left({ }^{*}\right)$, J. Merlin et J. Perez \\ I.N.S.A. de Lyon, G.E.M.P.P.M., U.A. 341, CALFETMAT, Bât. 403, 69621 Villeurbanne Cedex, France
}

(Reçu le 25 novembre 1986, accepté le 18 mars 1986)

\begin{abstract}
Résumé. - Plusieurs dispositifs susceptibles de conduire dans le cas de faisceau laser de forte puissance à une répartition énergétique rectangulaire et uniforme sont décrits. Tous ces dispositifs ont en commun la décomposition du faisceau primaire gaussien en 4 sous-faisceaux se recombinant au niveau de la zone d'interaction avec le matériau à « éclairer ». Une étude théorique détaillée de la répartition énergétique au niveau de cette zone d'interaction, a été réalisée de manière comparative afin de mettre en évidence les qualités de chaque dispositif et une analyse succincte de la facilité de mise en ouvre de ces dispositifs est abordée.

Abstract. - Several devices able to transform a Gaussian into a rectangular uniform high power laser beam, are described. With all these devices, the incident Gaussian beam is divided into 4 beams which are recombined at the level of the surface of the material interacting with the laser beam. The energy distribution in this region is theoretically analysed and a comparison between the different devices is made in order to mention the specific characteristics of each one, including the facilities of construction.
\end{abstract}

\section{Introduction.}

L'utilisation de sources laser pour la réalisation de traitements superficiels homogènes de matériaux, nécessite l'emploi de sources émettant en continu de fortes puissances, c'est-à-dire, pratiquement de laser $\mathrm{CO}_{2}$ continus multikilowatts émettant un rayonnement de longueur d'onde $\lambda=10,6 \mu \mathrm{m}$. Cela impose également d'avoir une densité énergétique uniforme au niveau de l'impact ainsi qu'un temps "d'éclairement " indépendant de la localisation dans la zone d'interaction faisceau-matériau. Il est donc indispensable dans le cas de cavités émettant un rayonnement ayant une symétrie axiale et une répartition sensiblement gaussienne (mode $\mathrm{TEM}_{00}$ ), de transformer cette répartition de manière à ce qu'elle soit sensiblement uniforme et de section carrée au niveau de l'impact avec le matériau.

Dans ce but plusieurs dispositifs ont déjà été proposés, voir commercialisés $[1,4]$. Nous avons nous même étudié un dispositif susceptible de répondre à ce problème [5]. L'objet du présent travail est de réaliser

$\left(^{*}\right)$ Adresse permanente : Institut de technologie de Kun Ming, Yunnam, République Populaire de Chine. pour ce dispositif « $\mathrm{L}_{1}$ », une étude plus détaillée de la répartition énergétique au niveau de la zone d'interaction avec le matériau et d'effectuer une analyse similaire d'une variante de ce système que nous décrirons plus loin (dispositif " $\mathrm{L}_{2}$ ") ainsi que du système proposé par Kawamura et al. [2] (dispositif « $\mathrm{K} »)$. Ces différents dispositifs présentent en effet des similitudes de principe et il nous est donc paru intéressant de dégager d'un point de vue théorique leurs avantages et inconvénients respectifs tout en esquissant les éléments de comparaison concernant leur facilité de mise en œuvre.

\section{Présentation générale des dispositifs.}

Pour les 3 dispositifs retenus, le faisceau laser gaussien incident de longueur d'onde $\lambda$ et de rayon $W$ se trouve scindé en 4 sous-faisceaux symétriques par rapport à l'axe optique du système, sous-faisceaux qui seront ensuite superposés et recombinés au niveau de la zone d'interaction avec le matériau. Ces 3 dispositifs diffèrent quant à la nature des éléments (miroirs ou dioptre) retenus pour réaliser la division du faisceau, ainsi que par leur disposition relative. 
2.1 DeSCRIPTION DU DISPOSITIF « $L_{1} »$. - Sur la figure la est représentée schématiquement ce dispositif composé d'un prisme réfléchissant à 4 faces également inclinées de $(\pi / 2+\alpha)$ par rapport à l'axe du système et réfléchissant le faisceau primaire sur 4 miroirs sphériques de rayon $2 f$ et situés à une distance $d_{0}$ des plans objets passant par le sommet du prisme et perpendiculaires à la direction des 4 sous-faisceaux réfléchis. Ces 4 sous-faisceaux sont ensuite focalisés par les miroirs sphériques et se recombinent ainsi à la distance $d_{\mathrm{i}}$ des miroirs aux niveaux des plans images; l'angle $\phi$ entre plans images et plan de superposition étant très faible.
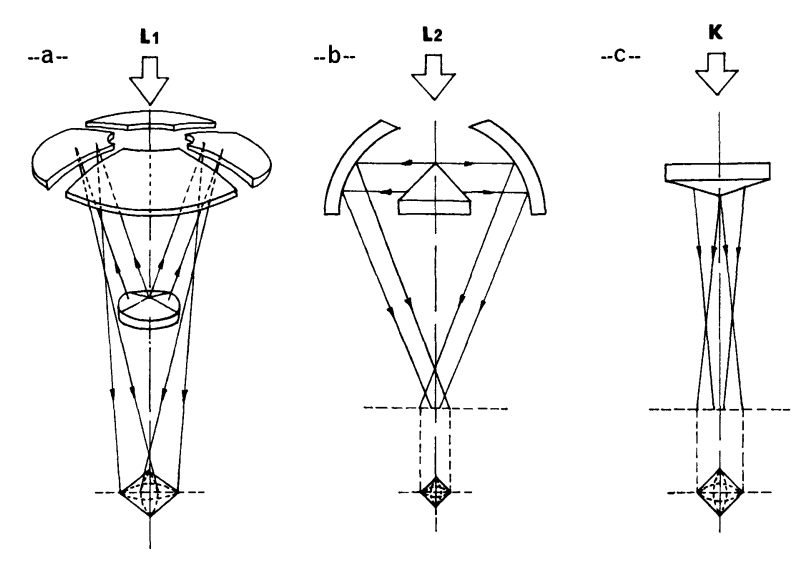

Fig. 1. - Schéma de principe des 3 dispositifs étudiés : a) dispositif « $L_{1}$ "; b) dispositif « $L_{2}$ "; c) dispositif « $K$ " (proposé par Kawamura et al. [2]).

[Schematic diagrams of the three studied devices : a) " $\mathrm{L}_{1}$ " device; b) « $\mathrm{L}_{2}$ " device; $c$ ) « $\mathrm{K}$ » device (proposed by Kawamura et al. [2]).]

Il a ainsi pu être montré [5] qu'à partir du moment où la condition :

$$
\frac{\lambda}{\sqrt{2} \sin \phi} \ll W
$$

est satisfaite, la répartition énergétique résultante peut s'exprimer par la somme des intensités des 4 sousfaisceaux et que cette répartition est sensiblement uniforme lorsque $2 L / M W=1,1$ (avec $2 L$ largeur de la tache après recombinaison et $M$ grandissement transversal.).

2.2 DesCRIPTION DU DISPOSITIF « $\mathrm{L}_{2}$ » (Fig. 1b). Le principe général de ce dispositif reste identique au précédent mais la disposition relative des divers éléments est différente. Les faces du prisme réfléchissant présentent une inclinaison plus importante et sont situées plus près des miroirs, à une distance $d_{0}$ bien inférieure à la distance focale $f$. Il en résulte l'impossibilité d'avoir une image réelle d'un plan objet défini comme pour le dispositif " $L_{1}$ "; on utilisera donc une simple recombinaison des 4 sous-faisceaux dans un plan quelconque situé à une distance $d_{\mathrm{i}}$ des miroirs sphériques.

Cette configuration permet d'avoir un dispositif beaucoup plus compact que celui précédemment étudié, mais nécessite un traitement théorique différent, certaines approximations n'étant plus valables.

2.3 DesCription DU DispositiF "K» (Fig. 1c). Dans ce dispositif les auteurs [2] proposent de réaliser la subdivision du faisceau au moyen d'un prisme à 4 faces, transparent au rayonnement incident. Du point de vue optique, ce dispositif est équivalent au dispositif « $L_{2}$ " dans le cas limite ou les miroirs sphériques sont de courbure infinie (donc remplacés par des miroirs plans).

Il en résulte que l'analyse théorique des répartitions énergétiques dans le cas du dispositif « $L_{2}$ " pourra être aisément transposée dans le cas de ce dispositif dioptrique.

\section{Etude de la répartition énergétique après recompo- sition des 4 sous-faisceaux.}

Nous étudierons dans ce paragraphe, à partir de la théorie de l'optique de Fourier, et en supposant que le

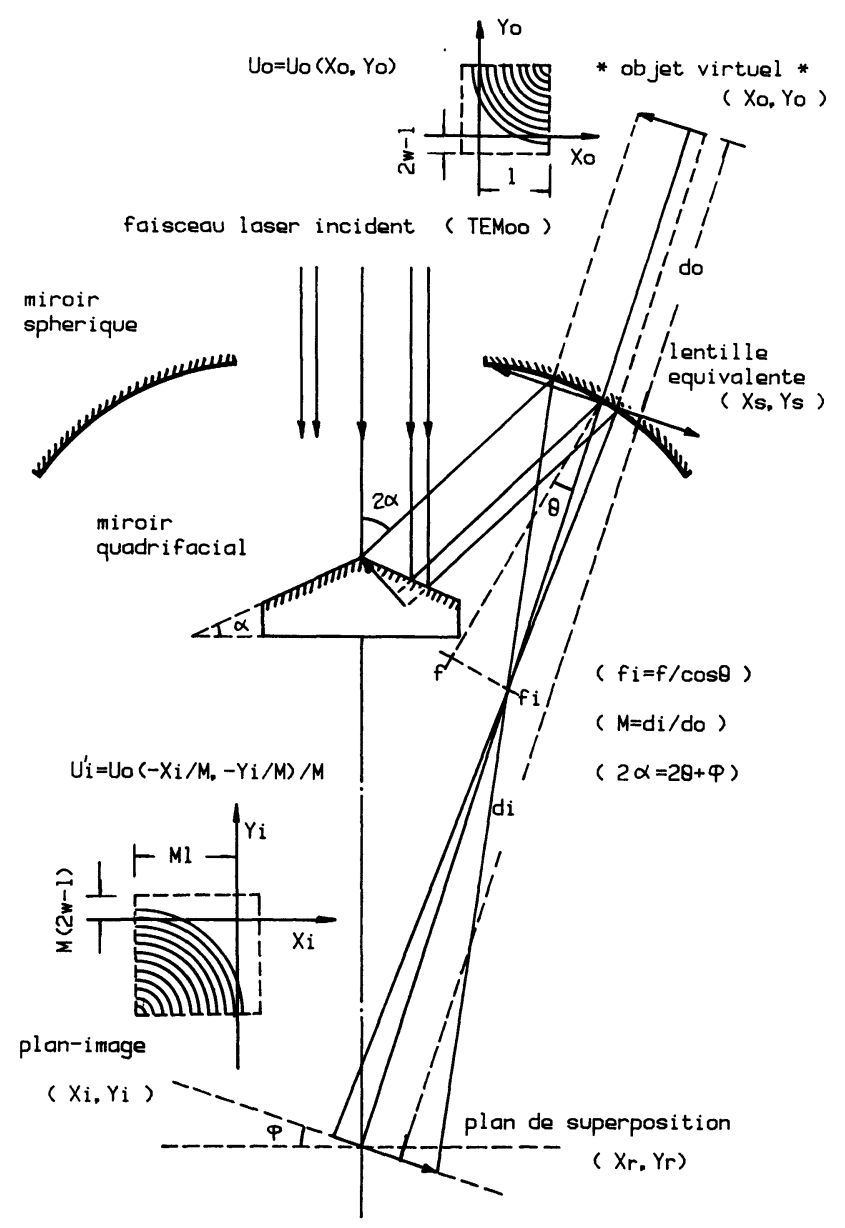

Fig. 2. - Représentation des chemins optiques équivalents pour les dispositifs étudiés.

[Equivalent optique paths for the studied devices.] 
faisceau laser incident est rigoureusement parallèle, la recombinaison des 4 sous-faisceaux au niveau de la zone d'impact et cela pour les situations correspondant au dispositif « $\mathrm{L}_{1}$ » et $« \mathrm{~L}_{2}$ » ou « $\mathrm{K} »$.

3.1 PRINCIPE DU CALCUL DANS LE CAS DU DISPOSITIF « $L_{1}$ ». - On peut considérer comme cela est schématisé sur la figure 2 qu'un miroir sphérique est équivalent à une lentille devant laquelle est situé un objet virtuel (face du prisme réfléchissant) situé à la distance $d_{0}$ à condition que l'angle d'incidence sur les miroirs sphériques soit suffisamment petit. Les chemins optiques du dispositif équivalent ainsi que le repérage des axes de coordonnées sont mentionnés sur la figure 2. L'angle entre le plan image de chaque sousfaisceau et le plan de superposition (plan perpendicu- laire au faisceau primaire) étant petit, on pourra considérer que la répartition d'énergie dans le plan image est identique à sa projection dans le plan de superposition.

D'après l'optique de Fourier, la fonction image $U_{\mathrm{i}}\left(X_{\mathrm{i}}, Y_{\mathrm{i}}\right)$ représentant la répartition dans le plan image est égale au produit de convolution entre la réponse impulsionnelle $n$ de la lentille équivalente et la fonction image prévue par l'optique géométrique [6].

On a :

$$
U_{\mathrm{i}}\left(X_{\mathrm{i}}, Y_{\mathrm{i}}\right)=h\left(X_{\mathrm{i}}, Y_{\mathrm{i}}\right) \otimes \frac{1}{M} U_{0}\left(-\frac{X_{\mathrm{i}}}{M},-\frac{Y_{\mathrm{i}}}{M}\right) .
$$

En faisant l'approximation selon laquelle les lentilles équivalentes sont de forme carrée de côté $2 a$, l'expression (1) devient :

$U_{\mathrm{i}}\left(X_{\mathrm{i}}, Y_{\mathrm{i}}\right)=\iint_{-M l}^{M(2 W-l)} \frac{\sin \frac{\Pi}{T}\left(X-X_{\mathrm{i}}\right)}{\Pi\left(X-X_{\mathrm{i}}\right)} \frac{\sin \frac{\Pi}{T}\left(Y-Y_{\mathrm{i}}\right)}{\Pi\left(Y-Y_{\mathrm{i}}\right)} \cdot \frac{1}{M} \exp \left[-\frac{(X+M l)^{2}+(Y+M l)^{2}}{M^{2} W^{2}}\right] \mathrm{d} X \mathrm{~d} Y$

avec $T=\frac{\lambda d_{\mathrm{i}}}{2 a}, d_{\mathrm{i}}$ distance image et $l=L / M$.

Cette expression (2) peut être exprimée par :

$$
U_{\mathrm{i}}\left(X_{\mathrm{i}}, Y_{\mathrm{i}}\right)=\frac{1}{M} U_{\mathrm{i}}\left(X_{\mathrm{i}}\right) \cdot U_{\mathrm{i}}\left(Y_{\mathrm{i}}\right)
$$

avec :

$$
U_{\mathrm{i}}\left(X_{\mathrm{i}}\right)=\int_{-M l}^{M(2 W-l)} \frac{\sin \frac{\Pi}{T}\left(X-X_{\mathrm{i}}\right)}{\Pi\left(X-X_{\mathrm{i}}\right)} \exp \left[-\frac{(X+M l)^{2}}{M^{2} W^{2}}\right] \mathrm{d} X
$$

et :

$$
U_{\mathrm{i}}\left(Y_{\mathrm{i}}\right)=\int_{-M l}^{M(2 W-l)} \frac{\sin \frac{\Pi}{T}\left(Y-Y_{\mathrm{i}}\right)}{\Pi\left(Y-Y_{\mathrm{i}}\right)} \exp \left[-\frac{(Y+M l)^{2}}{M^{2} W^{2}}\right] \mathrm{d} Y .
$$

L'énergie $I_{\mathrm{i}}\left(X_{\mathrm{i}}, Y_{\mathrm{i}}\right)$ au niveau d'un sous-faisceau est alors :

$$
I_{\mathrm{i}}\left(X_{\mathrm{i}}, Y_{\mathrm{i}}\right)=\frac{1}{M^{2}} U_{\mathrm{i}}^{2}\left(X_{\mathrm{i}}\right) \cdot U_{\mathrm{i}}^{2}\left(Y_{\mathrm{i}}\right)
$$

et l'énergie totale $I$ après superposition des 4 sous-faisceaux est :

$$
I=I_{\mathrm{i}}\left(X_{\mathrm{r}}, Y_{\mathrm{r}}\right)+I_{\mathrm{i}}\left(-X_{\mathrm{r}}, Y_{\mathrm{r}}\right)+I_{\mathrm{i}}\left(-X_{\mathrm{r}},-Y_{\mathrm{r}}\right)+I_{\mathrm{i}}\left(X_{\mathrm{r}},-Y_{\mathrm{r}}\right) .
$$

Il est ainsi possible de déterminer la répartition énergétique au niveau de la zone de superposition; toutefois il n'est pas possible d'exprimer ce résultat au moyen de fonctions analytiques simples et des résultats graphiques obtenus par calcul numérique seront fournis dans le paragraphe 4.

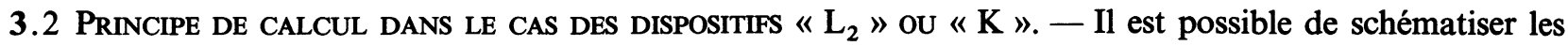
chemins optiques équivalents du dispositif « $\mathrm{L}_{2}$ » de manière analogue à ceux du dispositif « $\mathrm{L}_{1}$ » (voir Fig. 2). Cependant la distance objet : $d_{0}$ étant bien inférieure à la distance focale $f_{\mathrm{i}}$ des lentilles équivalentes, l'analyse ne peut se faire qu'à partir des approximations de Fresnel pour la diffraction. 
Comme $d_{0}$ est très petit, la répartition du champ sur la surface de la lentille équivalente peut être approchée par l'optique géométrique et après traversée de la lentille le champ devient [6] :

$$
U_{0}\left(X_{\mathrm{s}}, Y_{\mathrm{s}}\right)=\exp \left[j \frac{2 \pi}{\lambda} d_{0}\right] \cdot \exp \left[-\frac{\left(X_{\mathrm{s}}-l\right)^{2}+\left(Y_{\mathrm{s}}-l\right)^{2}}{W^{2}}\right] \exp \left[-j \frac{\pi}{\lambda f_{\mathrm{i}}}\left(X_{\mathrm{s}}^{2}+Y_{\mathrm{s}}^{2}\right)\right]
$$

avec

$$
\begin{aligned}
& -(2 w-l) \leqslant X_{\mathrm{s}} \leqslant l \\
& -(2 w-l) \leqslant Y_{\mathrm{s}} \leqslant l
\end{aligned} \quad \text { et } \quad f_{\mathrm{i}}=f / \cos \theta .
$$

Le champ dans un plan situé à une distance $d_{\mathrm{i}}$ de la lentille équivalente (c'est-à-dire des miroirs) est alors donné par :

$$
\begin{aligned}
U_{\mathrm{i}}\left(X_{\mathrm{i}}, Y_{\mathrm{i}}\right)=\frac{\exp \left[j \frac{2 \pi}{\lambda}\left(d_{\mathrm{i}}+d_{0}\right)\right]}{j \lambda d_{\mathrm{i}}} \iint_{-(2 W-l)}^{l} \exp \left[-\frac{\left(X_{1}-l\right)^{2}+\left(Y_{1}-l\right)^{2}}{W^{2}}\right] \times \\
\quad \times \exp \left[-j \frac{\pi}{\lambda f_{\mathrm{i}}}\left(X_{1}^{2}+Y_{1}^{2}\right)\right] \cdot \exp \left\{j \frac{\pi}{\lambda d_{\mathrm{i}}}\left[\left(X_{1}-X_{\mathrm{i}}\right)^{2}+\left(Y_{1}-Y_{\mathrm{i}}\right)^{2}\right]\right\} \mathrm{d} X_{1} \mathrm{~d} Y_{1} .
\end{aligned}
$$

L'expression (5) peut encore s'écrire :

$$
\begin{aligned}
U_{\mathrm{i}}\left(X_{\mathrm{i}}, Y_{\mathrm{i}}\right)=\frac{\exp \left\{j \frac{2 \pi}{\lambda}\left[d_{\mathrm{i}}+d_{0}+D\left(X_{\mathrm{i}}\right)+D\left(Y_{\mathrm{i}}\right)\right]\right\}}{j \lambda d_{\mathrm{i}} N^{2}} \iint_{-N l}^{N(2 W-l)} \exp \left[-\frac{(X+N l)^{2}+(Y+N l)^{2}}{N^{2} W^{2}}\right] \times \\
\times \exp \left\{j \frac{\pi}{S}\left[\left(X-X_{\mathrm{i}}\right)^{2}+\left(Y-Y_{\mathrm{i}}\right)^{2}\right]\right\} \mathrm{d} X \mathrm{~d} Y
\end{aligned}
$$

avec :

$$
\begin{gathered}
N=\frac{d_{\mathrm{i}}}{f_{\mathrm{i}}}-1 \\
S=-N d_{\mathrm{i}} \\
X=-N X_{1} \quad Y=-N Y_{1} \\
D\left(X_{\mathrm{i}}\right)=\frac{X_{\mathrm{i}}^{2}}{2 d_{\mathrm{i}}}\left(1+\frac{1}{N}\right), \quad D\left(Y_{\mathrm{i}}\right)=\frac{Y_{\mathrm{i}}^{2}}{2 d_{\mathrm{i}}}\left(1+\frac{1}{N}\right) .
\end{gathered}
$$

L'expression (6) montre que l'application de l'optique de Fourier à une lentille, conduit à un champ de diffraction, dans un plan situé à une distance $d_{\mathrm{i}}$, analogue à la diffraction de Fresnel dans un plan situé à une distance $N d_{\mathrm{i}}$, depuis la surface de cette lentille, pour un objet grandi transversalement de $N$ fois.

Ainsi lorsque $d_{\mathrm{i}}<f_{\mathrm{i}}$, on obtient un champ de diffraction direct et réduit $(|N|<1)$ et lorsque $d_{\mathrm{i}}>f_{\mathrm{i}}$ le champ de diffraction sera inversé mais agrandi de $N$ fois. Son grandissement transversal sera fourni par l'expression (7) qui permet de choisir les paramètres du dispositif selon les dimensions souhaitées pour la tache après recomposition des sous-faisceaux.

Il faut cependant remarquer que lorsque $d_{\mathrm{i}}=f_{\mathrm{i}}$, il est nécessaire de reprendre la discussion au niveau de l'expression (5). Dans cette situation les facteurs de phase au carré disparaissent et cette expression se réduit à la diffraction de Fraunhofer d'un quart du faisceau dans le plan focal.

L'énergie $I_{\mathrm{i}}\left(X_{\mathrm{i}}, Y_{\mathrm{i}}\right)$ au niveau d'un sous-faisceau s'exprime par :

$$
U_{\mathrm{i}}\left(X_{\mathrm{i}}, Y_{\mathrm{i}}\right) \cdot U_{\mathrm{i}}^{*}\left(X_{\mathrm{i}}, Y_{\mathrm{i}}\right)=\frac{1}{\lambda^{2} d_{\mathrm{i}}^{2} N^{4}}\left[A^{2}\left(X_{\mathrm{i}}\right)+B^{2}\left(X_{\mathrm{i}}\right)\right] \cdot\left[A^{2}\left(Y_{\mathrm{i}}\right)+B^{2}\left(Y_{\mathrm{i}}\right)\right]
$$

avec

$$
A\left(X_{\mathrm{i}}\right)=\int_{-N l}^{N(2 W-l)} \exp \left[-\frac{(X+N l)^{2}}{N^{2} W^{2}}\right] \cos \frac{\pi}{|S|}\left(X-X_{\mathrm{i}}\right)^{2} \mathrm{~d} X
$$




$$
\begin{aligned}
& B\left(X_{\mathrm{i}}\right)=\int_{-N l}^{N(2 W-l)} \exp \left[-\frac{(X+N l)^{2}}{N^{2} W^{2}}\right] \sin \frac{\pi}{|S|}\left(X-X_{\mathrm{i}}\right)^{2} \mathrm{~d} X \\
& A\left(Y_{\mathrm{i}}\right)=\int_{-N l}^{N(2 W-l)} \exp \left[-\frac{(Y+N l)^{2}}{N^{2} W^{2}}\right] \cos \frac{\pi}{|S|}\left(Y-Y_{\mathrm{i}}\right)^{2} \mathrm{~d} Y \\
& B\left(Y_{\mathrm{i}}\right)=\int_{-N l}^{N(2 W-l)} \exp \left[-\frac{(Y+N l)^{2}}{N^{2} W^{2}}\right] \sin \frac{\pi}{|S|}\left(Y-Y_{\mathrm{i}}\right)^{2} \mathrm{~d} Y
\end{aligned}
$$

Par raison de symétrie la répartition d'énergie après superposition des 4 sous-faisceaux s'exprime par une expression analogue à la relation (4) pour le dispositif $L_{1}$.

3.3 CoMparaison Des RÉSUltats OBTENUS PAR CALCUL NUMÉRIQUE POUR LES DEUX TYPES DE DISPOSITIFS. Compte tenu de l'équipement du laboratoire (sources
CILAS CI 1000 et CI 4000) nous nous sommes placés dans le cas d'une source laser $\mathrm{CO}_{2}$ continue $(\lambda=$ $10,6 \mu \mathrm{m}$ ) ayant un rayon de faisceau $W$ de $10 \mathrm{~mm}$

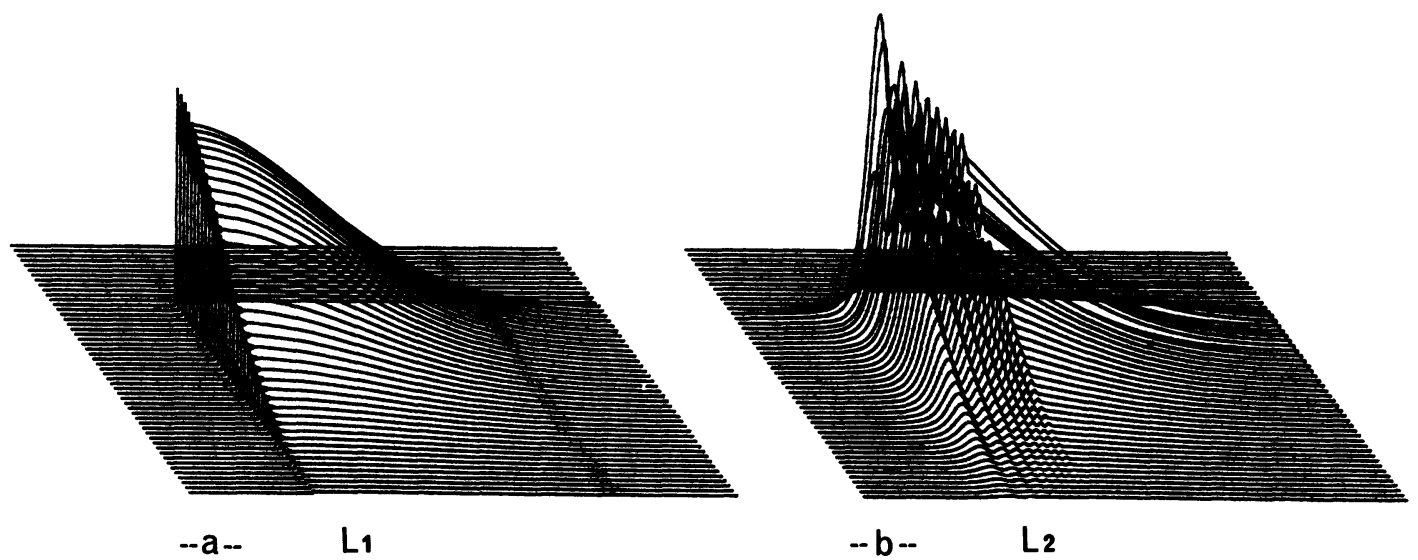

Fig. 3. - Exemple de répartition d'énergie obtenue au niveau du plan de superposition avant recombinaison : a) dispositif " $L_{1}$ »; b) dispositifs « $L_{2}$ " ou « $K$ ».

[Example of elementary beam intensity distribution in the recombination plane before recombination : a) « $\mathrm{L}_{1} »$ device; b) « $\mathrm{L}_{2}$ " or « $\mathrm{K}$ » devices.]

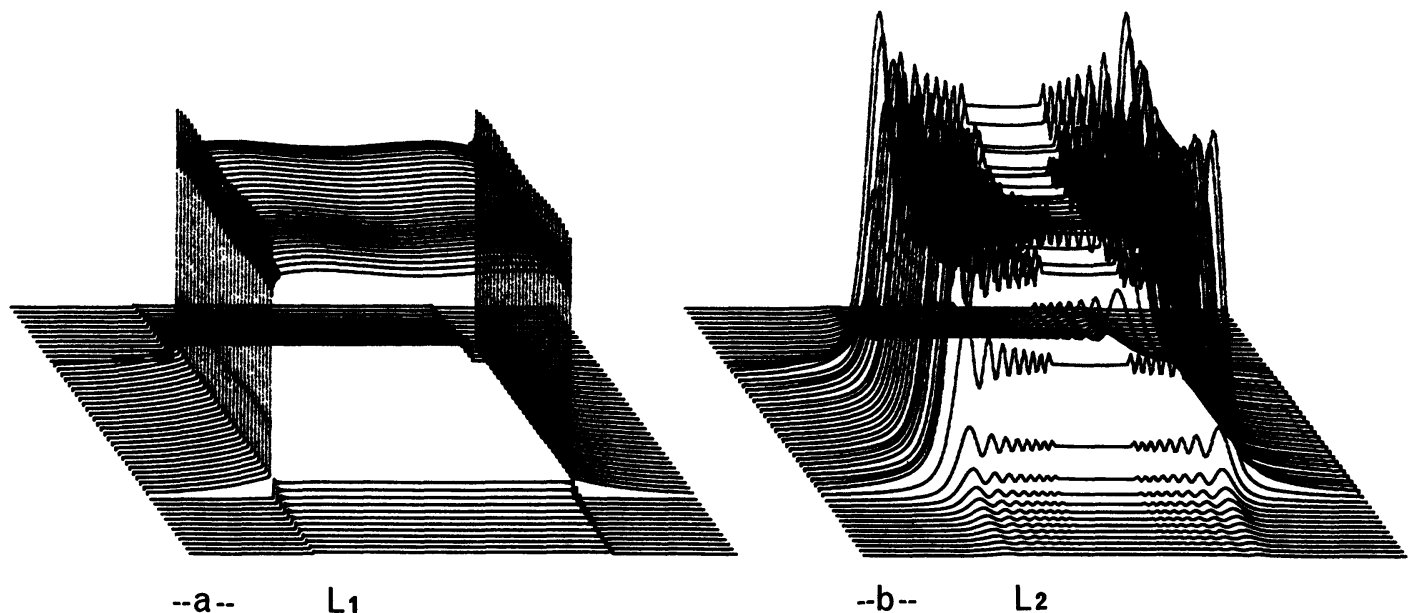

Fig. 4. - Répartition d'énergie obtenue dans le plan de superposition après recombinaison, avec les mêmes paramètres optiques que pour la figure 3.

[Intensity distribution in the recombination plane after recombination of the four elementary beams with the same optical parameters as in figure $3: a)$ « $L_{1}$ » device; b) « $L_{2}$ » or « $K$ » devices.] 
et nous avons évalué la répartition énergétique dans le plan de superposition avec les 2 types de dispositif dans le cas d'un grandissement transversal du champ $(M$ ou $N)$ de 1,5, c'est-à-dire de manière à avoir après recomposition une tache carrée de $16,5 \mathrm{~mm}$ de côté $(=1,1 \mathrm{MW})$.

Pour le dispositif $\mathrm{L}_{1}$ cela nécessite de prendre $2 a=100 \mathrm{~mm} d_{0}=200 \mathrm{~mm}$ et $d_{\mathrm{i}}=300 \mathrm{~mm}$; en ce qui concerne le dispositif $\mathrm{L}_{2}, d_{\mathrm{i}}$ doit être égale à $150 \mathrm{~mm}$.

Les répartitions d'énergie pour un sous-faisceau au niveau du plan de superposition sont données par les figures $3 a$ et $3 b$.

Les répartitions d'énergie après superposition des 4 sous-faisceaux toujours dans les mêmes conditions pour les différents dispositifs sont données par les figures $4 \mathrm{a}$ et $4 \mathrm{~b}$.

\section{Influence des différents paramètres sur la répartition d'énergie dans la zone d'interaction.}

Les résultats du paragraphe précédent semblent indiquer que le dispositif $\mathrm{L}_{1}$ conduit à une répartition d'énergie bien plus uniforme que les deux autres dispositifs. Il est cependant intéressant de réaliser une analyse moins subjective s'appuyant sur des grandeurs susceptibles d'influer sur la température localement atteinte en surface d'un matériau soumis à de telles répartitions d'énergie. Cette température tend en effet à être directement proportionnelle au flux d'énergie localement communiqué au matériau [7]. Si on schématise (Fig. 5) le profil idéal et celui correspondant à une répartition hypothétique, il apparaît que 3 paramètres sont principalement à prendre en compte :

- l'étalement d'énergie en bord de tache de largeur $2 L=2 M l$, c'est-à-dire la largeur $\Delta$ sur laquelle s'effectue la montée en énergie,

- la période $\Delta_{k}$ des fluctuations d'énergie autour de la valeur moyenne désirée $H$,

- l'amplitude $h$ des fluctuations les plus importantes ainsi que l'étendue de ces fluctuations vis-à-vis de la surface totale traitée.

Plus que les valeurs absolues de ces grandeurs ce sont d'ailleurs les valeurs relatives $\Delta / L$ et $h / H$ qui seront à évaluer et à minimiser pour avoir un dispositif le plus performant possible.

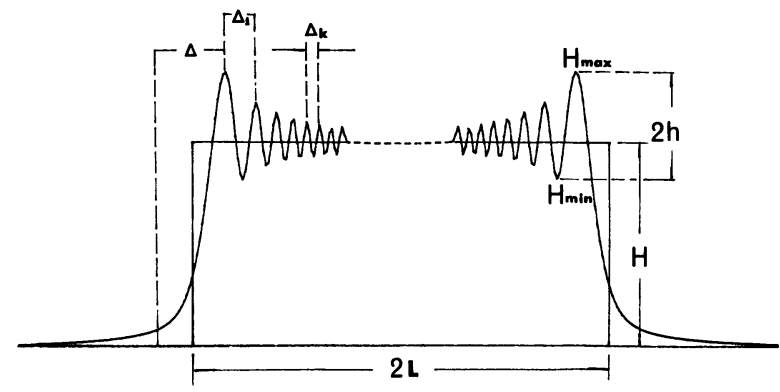

Fig. 5. - Coupe axiale schématique de la répartition d'énergie au niveau du plan de superposition, avec la définition des paramètres susceptibles de décrire la qualité de cette répartition.

[Schematic axial section of energy distribution in the recombination plane, with definition of parameters allowing the description of the quality of the distribution.]

Une analyse fine de la répartition énergétique des 2 types de dispositifs va donc être reprise avec cet objectif.

4.1 ANALYSE FINE DE LA RÉPARTITION ÉNERGÉTIQUE DANS LE CAS DU DISPOSITIF $L_{1}$. - Nous avons vu dans le paragraphe 3 (relation (3)) que la fonction image est le produit de 2 intégrales du même type :

$$
\int_{a}^{b} \exp \left[-\frac{(X+l)^{2}}{W^{2}}\right] \frac{\sin \frac{\pi}{T}\left(X-X_{\mathbf{i}}\right)}{\pi\left(X-X_{\mathbf{i}}\right)} \mathrm{d} X .
$$

L'intervalle d'intégration est déterminé par la zone où la fonction objet existe. Dans le cas général où la fonction objet est $f(X)$ on a :

$$
G\left(X_{\mathrm{i}}\right)=\int_{-\infty}^{\infty} f(X) \frac{\sin \frac{\pi}{T}\left(X-X_{\mathrm{i}}\right)}{\pi\left(X-X_{\mathrm{i}}\right)} \mathrm{d} X
$$

En posant :

$$
V=\frac{\sin \frac{\pi}{T}\left(X-X_{\mathbf{i}}\right)}{\pi\left(X-X_{\mathbf{i}}\right)}
$$

cette expression peut également s'écrire :

$$
G\left(X_{\mathrm{i}}\right)=\int_{X_{\mathrm{i}}-T}^{X_{1}+T} f(X) \cdot V \mathrm{~d} X+\sum_{n=1}^{\infty}\left[\int_{X_{1}+n T}^{X_{1}+(n+1) T} f(X) \cdot V \mathrm{~d} X+\int_{X_{1}-(n+1) T}^{X_{1}-n T} f(X) \cdot V \mathrm{~d} X\right]
$$

ou encore :

$$
G\left(X_{\mathrm{i}}\right)=\int_{X_{\mathrm{i}}-T}^{X_{1}+T} f(X) \cdot V \mathrm{~d} X+\sum_{n=1}^{\infty} \frac{(-1)^{n}}{\pi}\left[\int_{0}^{T} f\left(X_{\mathrm{i}}+n T+t\right) \frac{\sin \frac{\pi}{T} t}{n T+t} \mathrm{~d} t+\int_{0}^{T} f\left(X_{\mathrm{i}}-n T-t\right) \frac{\sin \frac{\pi}{T} t}{n T+t} \mathrm{~d} t\right]
$$


avec :

$$
0 \leqslant t \leqslant T
$$

Dans cette expression on voit que la valeur de $G\left(X_{\mathrm{i}}\right)$ est déterminé principalement par la valeur de la fonction $f(X)$ entre $X_{\mathrm{i}}-T$ et $X_{\mathrm{i}}+T$. Elle dépend faiblement des autres termes correspondant aux autres intervalles. En outre ces contributions à $G\left(X_{\mathrm{i}}\right)$ seront d'autant plus faibles que la distance $n T$ grandira et les alternances de signes tendront aussi à en atténuer les effets. Compte tenu de ces remarques on peut obtenir un résultat précis de cette intégration, comme montré sur la figure 5 pour $\lambda=10,6 \mu \mathrm{m}$, $a=50 \mathrm{~mm}$ et $d_{\mathrm{i}}=300 \mathrm{~mm}$ (rappelons que $T=$ $\left.\lambda d_{\mathrm{i}} / 2 a\right)$.

D'après la figure 6 la valeur de $G\left(X_{\mathrm{i}}\right)$ est l'intégrale du produit de $f(X)$ par $V$ entre les bornes définies par la partie hachurée. Il est également possible d'obtenir $G^{2}\left(X_{\mathrm{i}}\right)$ (Fig. 6b et $6 \mathrm{c}$ ).

Il ressort de cette analyse que les paramètres $\Delta$ et $\Delta_{k}$ valent respectivement $\simeq 3 / 2 T$ et $2 T$, donc restent toujours de l'ordre de grandeur de la longueur d'onde du rayonnement incident $(T=32 \mu \mathrm{m}$ avec les valeurs choisies ici). Par conséquent la montée en énergie et la période des fluctuations ne dépassera pas quelques $0,1 \%$ des dimensions de la tache reconstituée ce qui est insignifiant.

L'amplitude relative $h / H$ des fluctuations en bord de tâche ne dépasse pas $10 \%$ et ces fluctuations s'amortissent très vite ce qui fait que très rapidement elles n'excèdent pas $1 \%$ de la valeur moyenne recherchée. On peut donc estimer que $90 \%$ au moins de la zone d'interaction avec le matériau est soumise à un flux énergétique pratiquement constant. La qualité de ce dispositif est donc particulièrement remarquable.

4.2 ANALYSE FINE DANS LE CAS DES DISPOSITIFS « $\mathrm{L}_{2}$ » OU « $K$ ». - L'expression (9) donne la répartition d'énergie de la tache recomposée dans le cas de ces dispositifs. Les résultats des calculs numériques sont rassemblés sur la figure 7 pour $d_{i}=500 \mathrm{~mm}, W=$ $10 \mathrm{~mm}, M=1,5, \lambda=10,6 \mu \mathrm{m}$ et $2 L / M W=1,1$.

Pour discuter ces résultats nous avons également

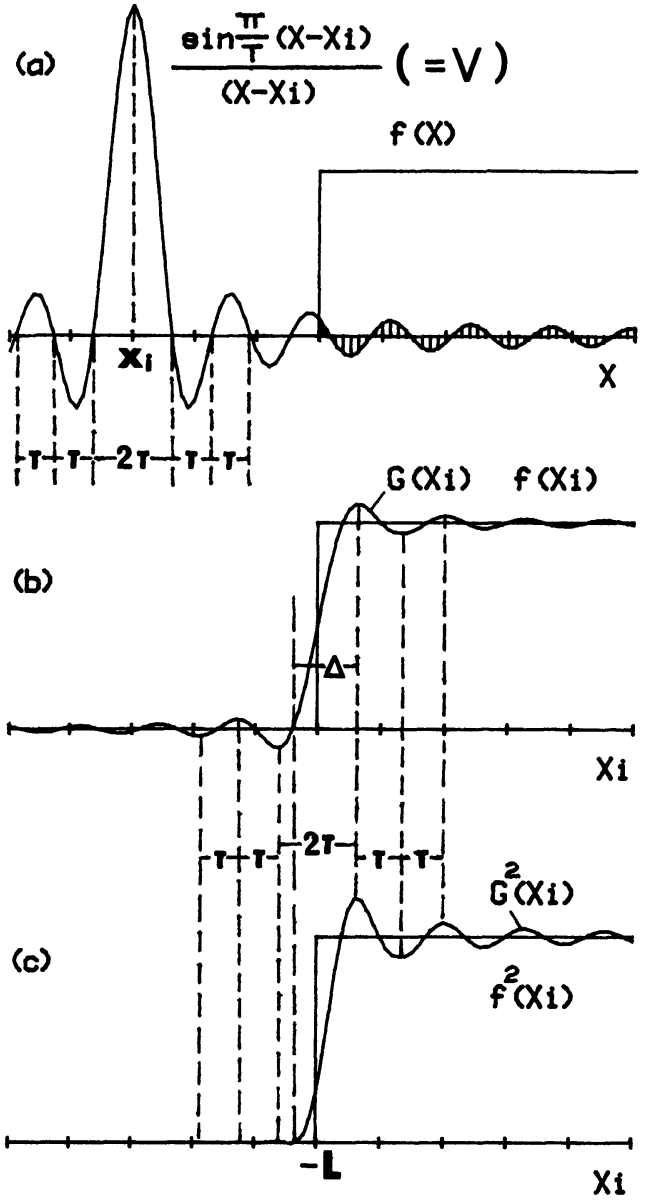

Fig. 6. - Représentation graphique des différentes fonctions mathématiques permettant d'accéder à la répartition d'énergie dans le cas du dispositif « $\mathrm{L}_{1}$ » : a) fonction « $V$ »; b) fonction $G\left(X_{\mathrm{i}}\right)$; c) fonction $G^{2}\left(X_{\mathrm{i}}\right)$.

[Graphic representation of mathematical functions necessary to describe the energy distribution for $« \mathrm{~L}_{1}$ " device : a) $V$ function; b) $G\left(X_{\mathfrak{i}}\right)$ function; c) $G^{2}\left(X_{\mathfrak{i}}\right)$ function.]

représenté sur cette figure les variations des fonctions sinus et cosinus de la fonction $\left(X-X_{\mathrm{i}}\right)^{2}$. Puis en utilisant la même méthode que précédemment nous avons décomposé les intégrales $A\left(X_{\mathrm{i}}\right)$ et $B\left(X_{\mathrm{i}}\right)$ de la façon suivante :

$$
\begin{gathered}
A\left(X_{\mathrm{i}}\right)=\int_{X_{1}-\sqrt{|S| / 2}}^{X_{1}+\sqrt{|S| / 2}} f(X) \cdot \cos Z \mathrm{~d} X+\sum_{n=1}^{\infty}\left[\int_{X_{1}+\sqrt{(2 n-1)|S| / 2}}^{X_{1}+\sqrt{(2 n+1)|S| / 2}} f(X) \cdot \cos Z \mathrm{~d} X+\right. \\
\left.+\int_{X_{1}-\sqrt{(2 n+1)|S| / 2}}^{X_{1}-\sqrt{(2 n-1)|S| / 2}} f(X) \cdot \cos Z \mathrm{~d} X\right] \\
B\left(X_{\mathrm{i}}\right)=\sum_{n=0}^{\infty}\left[\int_{X_{\mathrm{i}}+\sqrt{n|S|}}^{X_{\mathrm{i}}+\sqrt{(n+1)|S|}} f(X) \cdot \sin Z \mathrm{~d} X+\int_{X_{1}-\sqrt{(n+1)|S|}}^{X_{\mathrm{i}}-\sqrt{n|S|}} f(X) \cdot \sin Z \mathrm{~d} X\right]
\end{gathered}
$$




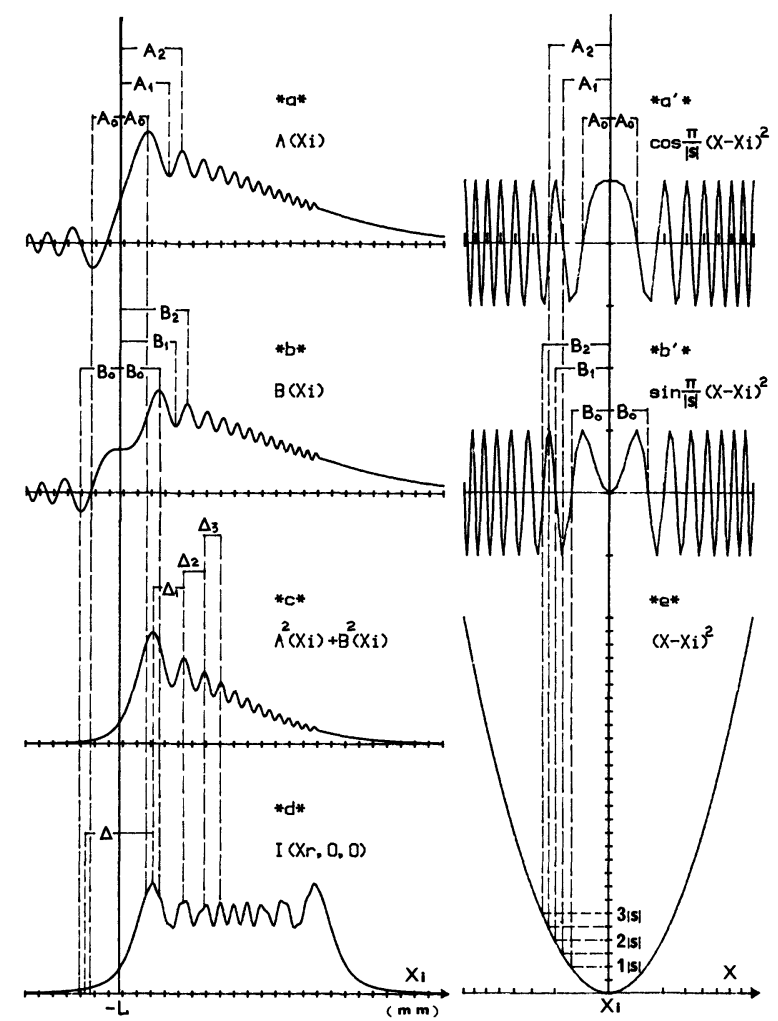

Fig. 7. - Représentation graphique des différentes fonctions mathématiques permettant d'accéder à la répartition d'énergie dans le cas des dispositifs « $\mathrm{L}_{2}$ » ou « $\mathrm{K}$ » : a) fonction $\left.A\left(X_{\mathrm{i}}\right) ; \mathrm{a}^{\prime}\right)$ fonction $\left.\cos Z ; \mathrm{b}\right)$ fonction $B\left(X_{\mathrm{i}}\right)$; b) fonction $\sin Z$; c) $A^{2}\left(X_{\mathrm{i}}\right)+B^{2}\left(X_{\mathrm{i}}\right)$ avant sommation des 4 sous-faisceaux; d) répartition d'énergie $I$ après sommation.

[Graphic representation of mathematical functions necessary to describe the energy distribution for $" \mathrm{~L}_{2}$ " or « $\mathrm{K}$ » devices. a) $A\left(X_{\mathrm{i}}\right)$ function; a') $\cos Z$ function; b) $B\left(X_{\mathrm{i}}\right)$ function; $\left.\mathrm{b}^{\prime}\right) \sin Z$ function; c) $A^{2}\left(X_{\mathrm{i}}\right)+B^{2}\left(X_{\mathrm{i}}\right)$ before beams recombination; d) intensity distribution $I$ after beams recombination.]

avec :

$$
Z=\frac{\pi}{|S|}\left(X-X_{\mathrm{i}}\right)^{2}
$$

La comparaison des expressions (11) et (12) (Fig. 7) avec l'expression (10) (Fig. 6) indique que les fonctions $\sin Z$ et $\cos Z$ possèdent des caractéristiques proches de la fonction $V$. Ces fonctions sont symétriques par rapport à l'axe $X=X_{\mathrm{i}}$, toutefois lorsque l'on s'éloigne de $X_{\mathrm{i}}$ l'amplitude des 2 premières reste constante alors que leur période diminue tandis que pour $V$ l'amplitude décroît graduellement avec une période invariable.

Pour le calcul des intégrales $A\left(X_{\mathrm{i}}\right)$ et $B\left(X_{\mathrm{i}}\right)$ après avoir procédé de la même façon que pour $G\left(X_{\mathrm{i}}\right)$ au paragraphe précédent, on obtient les paramètres suivants :
- Figure 7a :

$$
A_{k}=\sqrt{(2 k+1)|S| / 2} \quad k=0,1,2, \ldots
$$

- Figure $7 b$ :

$$
B_{k}=\sqrt{(k+1)|S|} \quad k=0,1,2, \ldots
$$

Sur les figures $7 \mathrm{c}$ et $7 \mathrm{~d}$ sont représentées les répartitions d'énergie avant et après superposition des sous-faisceaux.

Les paramètres $\Delta$ et $\Delta_{k}$ s'expriment respectivement par $A_{0}+B_{0}$ et $\left[\left(A_{2 k}+B_{2 k}\right)-\left(A_{2 k-2}+B_{2 k-2}\right)\right] / 2$ par conséquent sont toujours de l'ordre de grandeur de $\sqrt{|S|}$ avec $|S|=\left|N d_{\mathrm{i}}\right| \lambda, N$ étant le grandissement transversal du champ de diffraction qui reste toujours voisin de $1, S$ sera toujours de l'ordre de $1 \mathrm{~mm}^{2}$ et $\Delta, \Delta_{k}$ seront de l'ordre de $1 \mathrm{~mm}$. Soit 5 à $10 \%$ des dimensions de la tâche, c'èst-à-dire 1 à 2 ordre de grandeurs supérieurs aux mêmes caractéristiques dans le cas du dispositif $\mathrm{L}_{1}$. L'amélioration des performances des dispositifs $L_{2}$ ou $K$ passe donc par une réduction des valeurs de ces paramètres qui ne pourra être obtenu que par une réduction de la distance $d_{\mathbf{i}}$.

L'amplitude relative $h / H$ des plus grandes fluctuations atteint environ $25 \%$ ce qui est beaucoup, d'autant que ces fluctuations s'affaiblissent assez peu et au centre elles possèdent encore une amplitude de l'ordre de $10 \%$ par rapport au niveau moyen.

Cette amplitude de fluctuations assez marquée, associée à une périodicité de pas assez élevée est susceptible de poser problème pour les faibles temps d'interaction $\tau$ avec le matériau et cela sera d'autant plus critique que les matériaux possèderont une diffusivité thermique $\alpha$ faible. Des gradients de température pourront en effet subsister dans la zone d'interaction dès que (d'après [7]) $\Delta_{k} \simeq \sqrt{\alpha \tau}$, soit $\tau \simeq 1 / 10 \mathrm{~s}$ pour un alliage ferreux par exemple. Remarquons toutefois que la double périodicité en $X$ et en $Y$ atténuera ce problème dans le cas de traitements réalisés par balayage de la surface du matériau par le faisceau laser reconstitué.

\subsection{ETUde COMPARATIVE DES FLUCTUATIONS DE LA} RÉPARTITION ÉNERGÉTIQUE POUR LES DIFFÉRENTS DISPOSITIFS. - Afin de matérialiser les avantages et les inconvénients des différents dispositifs vis-à-vis des fluctuations de répartition, sur la figure 8 ont été rassemblés différents profils correspondant à une coupe transversale selon l'axe de la tache reconstituée et correspondant aux situations suivantes :

- dans tous les cas faisceau incident $(\lambda=10,6 \mu \mathrm{m})$, cylindrique à répartition gaussienne de rayon $W=$ $10 \mathrm{~mm}$ (le profil correspondant est matérialisé sur la figure); 


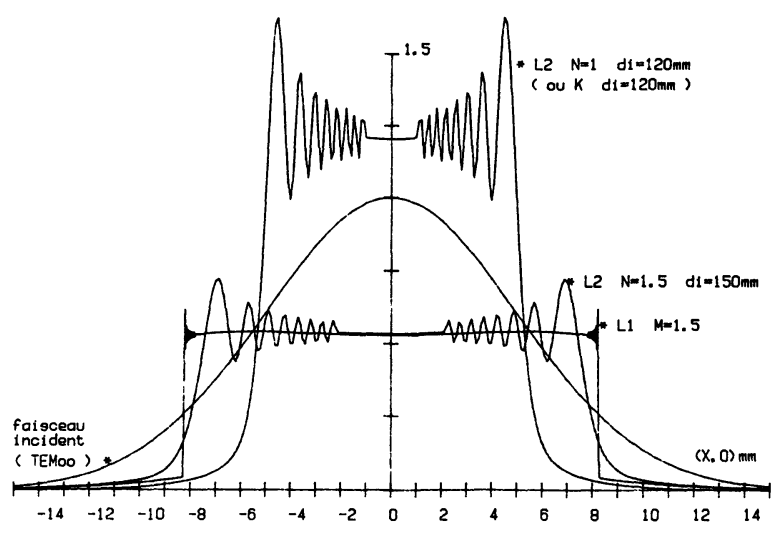

Fig. 8. - Coupe axiale de la répartition d'énergie dans le plan de superposition pour les 3 dispositifs avec différents couples de paramètres optiques.

[Axial section of energy distribution in the recombination plane for the three devices with several values of optical parameters.]

- pour un grandissement transversal $\mathbf{M}$ ou $\mathbf{N}$ de 1,5 les profils sont présentés pour les 2 dispositifs $\mathrm{L}_{1}$ $\left(f_{\mathrm{i}}=120 \mathrm{~mm}, d_{0}=200 \mathrm{~mm}, d_{\mathrm{i}}=300 \mathrm{~mm}\right)$ et $\mathrm{L}_{2}$ $\left(f_{\mathrm{i}}=60 \mathrm{~mm}, d_{\mathrm{i}}=150 \mathrm{~mm}\right)$. Noter que la finesse de la périodicité des fluctuations dans le cas du dispositif $\mathrm{L}_{1}$ interdit qu'on puisse les distinguer à l'échelle de la figure, et on remarque que l'on est excessivement proche du profil théorique obtenu à partir de la seule optique géométrique;

- enfin pour un grandissement de 1 a été présenté le profil de répartition correspondant, soit au dispositif $\mathrm{L}_{2}$ toujours avec des miroirs de focales $60 \mathrm{~mm}$ mais en se plaçant cette fois-ci à une distance $d_{\mathrm{i}}$ de $120 \mathrm{~mm}$, soit au dispositif $\mathrm{K}$ en se plaçant à la même distance $d_{\mathrm{i}}=120 \mathrm{~mm}$.

Les qualités de la répartition énergétique du dispositif $\mathrm{L}_{1}$ vis-à-vis des 2 autres sont incontestablement mises en évidence. Il faut par contre remarquer la possibilité de modifier la taille de la tache reconstituée dans le cas du dispositif $L_{2}$ et cela sans changement des éléments optiques. Enfin, il faut noter l'impossibilité de modification de cette taille $\left(f_{\mathrm{i}}=\infty, N=-1\right.$ obligatoirement) dans le cas de l'utilisation du dispositif $\mathrm{K}$.

\section{Conclusion.}

L'obtention d'une répartition énergétique sensiblement uniforme après subdivision d'un faisceau gaussien en 4 sous-faisceaux et recombinaison de ces sous-faisceaux au niveau de la zone d'utilisation est donc possible de différentes manières et nous avons plus particulièrement étudié 3 situations correspondant aux dispositifs que nous avons désignés $L_{1}, L_{2}$ et $K$.

Le dispositif $L_{1}$ constitué d'un prisme réfléchissant et de 4 miroirs sphériques disposés de telle manière que la zone d'interaction avec le matériau corresponde aux plans images des 4 sous-faisceaux, conduit à une répartition énergétique bien supérieure à celle des autres dispositifs. Le problème pour ce dispositif réside toutefois en son encombrement (plusieurs centaines de millimètres sont nécessaires entre les miroirs et la zone de recombinaison) et certainement dans les difficultés de réalisation et de réglages des différents éléments optiques.

Le dispositif $L_{2}$ constitué par des éléments optiques similaires à $\mathrm{L}_{1}$, mais beaucoup plus proches les uns des autres a donc un encombrement bien plus réduit que le précédent, des latitudes de réglages des éléments certainement bien plus grandes avec en particulier la possibilité de jouer sur les dimensions de la tache reconstituée mais en contre partie on aboutit à une répartition énergétique nettement moins uniforme.

Le dispositif $\mathrm{K}$ a l'avantage de présenter une très grande simplicité du point de vue optique puisqu'il n'est constitué que d'un prisme transparent, il présente par contre l'inconvénient de ne pas pouvoir jouer sur les dimensions de la tache reconstituée et d'avoir une répartition énergétique similaire à $L_{2}$ donc présentant des fluctuations marquées.

Par conséquent selon l'application envisagée il sera peut-être préférable de choisir tel ou tel dispositif, le dispositif $\mathrm{L}_{2}$ étant toutefois le dispositif présentant la plus grande souplesse d'utilisation.

Pour préciser ce choix une suite théorique à ce travail est actuellement en cours afin de simuler les réponses des dispositifs $\mathrm{L}_{1}$ et $\mathrm{L}_{2}$ à certaines anomalies inévitables telles que des variations de la position du plan de recombinaison des faisceaux. En outre un appareillage est en cours de montage afin de tester expérimentalement les possibilités réelles des dispositifs $L_{1}$ et $L_{2}$ en présence d'un faisceau et d'éléments optiques présentant obligatoirement des imperfections.

\section{Bibliographie}

[1] Shafer, D., Opt. Laser Technol. (1982) 159.

[2] Kawamura, Y., Itagaki, Y., Toyoda, K., Namba, S., Opt. Commun. 48 (1983) 44.

[3] DubroeucQ, G., Lacombat, M., Brevignon, M., U.S. Patent, 4, 370, 026 (1983).

[4] Dagenais, D. M., Woodroffe, J. A., Itzkan, I., Appl. Optics 24 (1985) 671.
[5] Girardeau-Montaut, J. P., Li, J. C., GirardeauMontaut, C., Optics Commun. (1986) (sous presse).

[6] Goodman, J. N., Introduction to Fourier Optics (McGraw Hill Book Cy N. Y.) 1968.

[7] Carlslaw, H. S., Jaeger, J. C., Conduction of Heat in solids (Oxford Press, 2nd ed) 1954. 